\title{
Disorder effects in the quantum Hall effect of graphene $\boldsymbol{p}$ - $\boldsymbol{n}$ junctions
}

\author{
Jian Li and Shun-Qing Shen \\ Department of Physics and Center for Theoretical and Computational Physics, The University of Hong Kong, \\ Pokfulam Road, Hong Kong, China
}

(Received 29 May 2008; revised manuscript received 1 October 2008; published 7 November 2008)

\begin{abstract}
The quantum Hall effect in graphene $p$ - $n$ junctions is studied numerically with emphasis on the effect of disorder at the interface of two adjacent regions. Conductance plateaus are found to be attached to the intensity of the disorder and are accompanied by universal conductance fluctuations in the bipolar regime, which is in good agreement with theoretical predictions of the random matrix theory on quantum chaotic cavities. The calculated Fano factors can be used in an experimental identification of the underlying transport character.
\end{abstract}

DOI: 10.1103/PhysRevB.78.205308

PACS number(s): 73.43.-f, 72.80.-r

\section{INTRODUCTION}

When a monolayer of honeycomb lattice is singled out of graphite, ${ }^{1}$ this two-dimensional material, dubbed graphene, acquires extraordinary electronic properties. ${ }^{2-4}$ Electrons in graphene mimic massless Dirac fermions with extremely high mobility and tunability, ${ }^{5,6}$ which makes this material interesting both theoretically and practically. The tunability of the carrier type via the electric-field effect, in particular, allows for the realization of graphene $p-n$ junctions using only electrostatic gating. ${ }^{7,8}$ The quantum Hall effect in these graphene $p$ - $n$ junctions has shown new fractional plateaus in the bipolar regime ${ }^{7}$ that were explained by uniform mixing among edge states at the junction interface. ${ }^{9}$ The mechanism of the mode mixing, however, is still unclear.

In this paper we address this problem by investigating the transport characteristics of graphene $p$ - $n$ junctions in the quantum Hall regime with disorder at the junction interface. Our calculations are based on the Landauer-Büttiker formalism for coherent transport, ${ }^{10}$ and the results are explained using the random matrix theory (RMT) of quantum transport. ${ }^{11,12}$

\section{MODEL AND METHOD}

The setup of our simulation is illustrated in Fig. 1. A graphene strip of width $W$ is divided into two regions by a transition area with length $d$. In either region the carrier type and density can be locally tuned through an electrostatic gating, and the relative value of the Fermi energy to the local charge neutrality point is defined as the relative Fermi energy $E_{f}^{(r)}$. The whole sample is subjected to a perpendicular magnetic field and the Landau levels are formed in the quantum Hall regime. ${ }^{13}$ With a specific Fermi energy, the filling factor of Landau levels is scarcely dependent on the details of the sample edge provided that the sample size is big enough; $;{ }^{14}$ therefore, we use samples with zigzag edges to carry out our simulation but the results are applicable to general cases. On the energy scale of our problem, both Zeeman splitting and spin-orbit interaction are negligibly small. Different spin states can be taken as degenerate and uncorrelated; thus, we assume that spin is irrelevant in our calculation and simply multiply the result by a factor accounting for the spin degree of freedom. For this reason the filling factors $\nu_{1}$ and $\nu_{2}$ given below are all "spinless," i.e., their values are $\pm 1, \pm 3, \pm 5 \ldots$ instead of $\pm 2, \pm 6, \pm 10 \ldots$.

Magnetoconductance of a graphene $p$ - $n$ junction has been theoretically discussed in terms of its valley isospin dependence. ${ }^{15}$ These discussions in general assume an absence of intervalley scattering across the graphene $p$ - $n$ junction. To explain the fractionally quantized plateaus observed in the experiments, ${ }^{7,8}$ however, full mode mixing was required in random matrix theory, which inevitably involves intervalley scattering. ${ }^{9}$ We attribute the intervalley scattering in our model to disorder potential, which varies quickly enough in the scale of the lattice constant. Disorder in graphene may have various sources, and the understanding of its role in transport properties is still incomplete., ${ }^{4,16}$

In this paper we focus on the effect of the random potential at the interface of the junction. The reason is that current inside either region 1 or region 2 is carried by quantum Hall edge states ${ }^{17}$ thus immune to most disorder inside either region, while scattering among states at the interface of the two regions contributes to the major effect of disorder on the overall conductance. Disorder at the interface may come from intrinsic sources such as vacancies and impurities or from extrinsic sources such as random potential introduced by the irregularities of the gate edge. We model the disorder potential at the interface by using the Anderson-type on-site energy ${ }_{18}^{18}$ which varies randomly from site to site within the transition area, where a potential slope connecting two sides

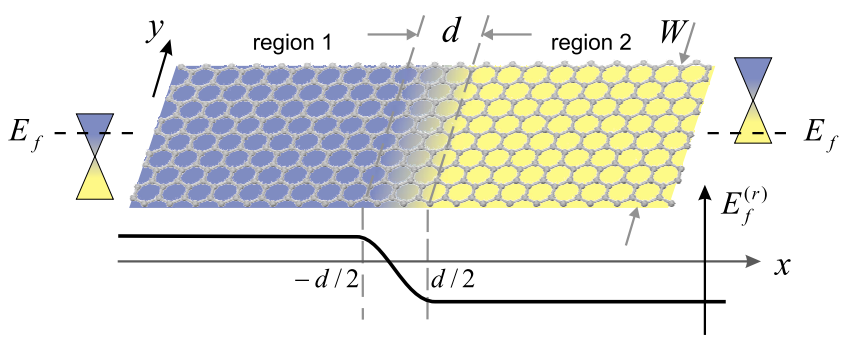

FIG. 1. (Color online) Schematic of a graphene junction. Two locally gate-controlled regions of a graphene strip (width $W$ ), connected with a reservoir at each of the far ends, are jointed by a transition area (length $d$ ) where potential is assumed to be composed of a slope along the strip and a random distribution over each site within. The lower curve shows the profile of the relative Fermi energy $E_{f}^{(r)}$. 
of the junction serves as the background potential. Hence the total Hamiltonian reads

$$
H=\sum_{i} \varepsilon\left(\boldsymbol{r}_{i}\right) c_{i}^{\dagger} c_{i}-\sum_{\langle i, j\rangle}\left[t e^{i \phi_{i j}} c_{i}^{\dagger} c_{j}+\text { H.c. }\right]
$$

where $c_{i}^{\dagger}$ and $c_{i}$ are the electron creation and annihilation operators at site $\boldsymbol{r}_{i} \equiv\left(x_{i}, y_{i}\right)$, respectively, $t \approx 2.8 \mathrm{eV}$ is the nearest-neighbor hopping energy in the graphene lattice, and $\langle i, j\rangle$ stands for a nearest-neighboring pair;

$$
\phi_{i j}=\frac{e}{\hbar} \boldsymbol{A}\left(\frac{\boldsymbol{r}_{i}+\boldsymbol{r}_{j}}{2}\right) \cdot\left(\boldsymbol{r}_{i}-\boldsymbol{r}_{j}\right)
$$

is the phase acquired from an electron hopping from $\boldsymbol{r}_{j}$ to $\boldsymbol{r}_{i}$ in an external field $B$ described by vector potential $A$ and the on-site energy $\varepsilon\left(\boldsymbol{r}_{i}\right)$ is $\varepsilon_{1}$ and $\varepsilon_{2}$ in regions 1 and 2 , respectively;

$$
\varepsilon\left(\boldsymbol{r}_{i}\right)=\left(\varepsilon_{2}-\varepsilon_{1}\right) \frac{x_{i}}{d}+\frac{1}{2}\left(\varepsilon_{1}+\varepsilon_{2}\right)+\operatorname{rand}(\Delta)
$$

in the transition area $\left(\left|x_{i}\right| \leq d / 2\right)$ with $\operatorname{rand}(\Delta)$ as a random number uniformly distributed in $[-\Delta / 2, \Delta / 2]$. The width of the junction in our simulation is taken to be $W$ $=200(\sqrt{3} a / 2) \approx 42.6 \mathrm{~nm}$, where $a \approx 0.246 \mathrm{~nm}$ is the lattice constant of graphene and the length of the interface area is taken to be $d=20 a \approx 4.9 \mathrm{~nm}$. Landau gauge $A(\boldsymbol{r})=(-B y, 0)$ is adopted with the magnitude of the magnetic field $B$ $\approx 113 \mathrm{~T}$, which is equivalent to a magnetic flux $\Phi$ $=h / 701 e$ in each unit cell. The magnetic length in this case is $l_{B}=\sqrt{\hbar /(e B)} \approx 2.4 \mathrm{~nm}$, which is about a half of the length of the interface area or $1 / 18$ of the width of the sample. It should be mentioned here that in a real sample, which is presumably much larger in size, the magnetic field necessary for the quantum Hall effect can be much smaller.

We assume coherent transport in the graphene junction, where the Landauer-Büttiker formalism can be applied. ${ }^{10}$ Transmission functions $T_{p q}(p, q=1,2$ and $p \neq q)$ of the junction are calculated by using the recursive Green's function technique. ${ }^{19,20}$ Vanishing net current in equilibrium implies that $T_{21}=T_{12}=T$; therefore, the conductance is proportional to either of the transmission functions $G=\left(e^{2} / h\right) T$, where the spin degeneracy has been included in $T$, or the variance of the conductance $\operatorname{Var}(G)=\left(e^{2} / h\right)^{2} \operatorname{Var}(T)$, where $\operatorname{Var}(T)$ represents the variance of $T$. In the following we will be satisfied with observing only the behavior of $T$ in different situations. Each situation, that is, each experimental condition under which measurements are made, is identified with a specified combination of $\varepsilon_{1}, \varepsilon_{2}$, and $\Delta$, while various configurations of disorder are subjected to some self-averaging process in each measurement. This self-averaging process could be a result of time-dependent electric field used in the experiments ${ }^{9}$ and will suppress the fluctuation of the measured conductance; thus it makes the mean value a reasonable account for the experimental observation. Our calculation extracts the mean and the variance of the transmission functions $T$ in each situation from output of 40000 samples with different disorder configurations.

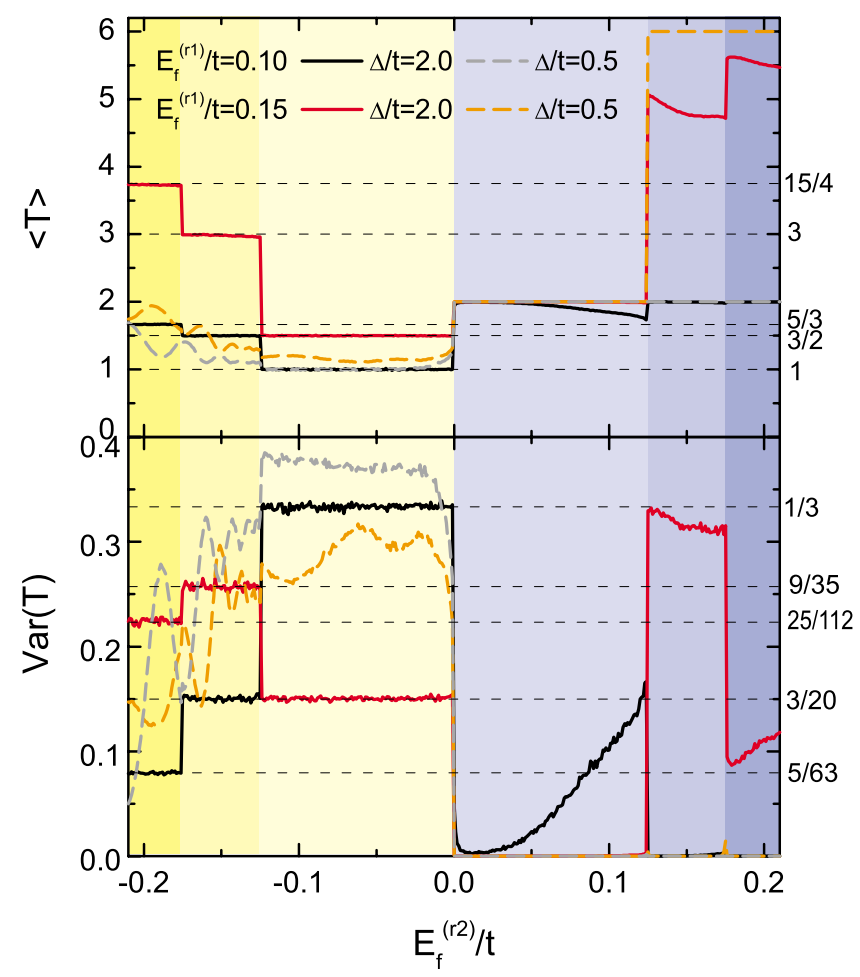

FIG. 2. (Color online) (a) The mean and (b) the variance of the transmission function $T$ as functions of the relative Fermi energy $E_{f}^{(r 2)}$ in region 2 with fixed relative Fermi energy $E_{f}^{(r 1)}$ in region 1 and fixed disorder strength $\Delta$. Cases with different $E_{f}^{(r 1)}$,s and different $\Delta$ 's are also compared. $E_{f}^{(r 1)}>0$ implies $n$ type of region 1 . $E_{f}^{(r 1)} / t=0.10$ corresponds to the filling factor $\nu_{1}=1$ and $E_{f}^{(r 1)} / t$ $=0.15$ corresponds to $\nu_{1}=3$.

\section{RESULTS}

The calculated transmission functions as shown in Fig. 2(a) have surprisingly recovered the quantized transport plateaus observed in the experiment by Williams et al. ${ }^{7}$ In junctions with the disorder strength $\Delta=2 t$ (solid lines in Fig. 2), the ensemble average of the transmission functions form nearly perfect plateaus in the bipolar regime $\left(E_{f}^{(r 2)}<0\right)$, and the height of each plateau is

$$
\langle T\rangle=2 \frac{\left|\nu_{1} \nu_{2}\right|}{\left|\nu_{1}\right|+\left|\nu_{2}\right|}
$$

with $\nu_{\text {total }} \equiv\left|\nu_{1}\right|+\left|\nu_{2}\right|$. Corresponding to each plateau of the averaged transmission function, the ensemble variance of $T$ also develops into a plateau described by

$$
\operatorname{Var}(T)=4 \frac{\left(\nu_{1} \nu_{2}\right)^{2}}{\left(\left|\nu_{1}\right|+\left|\nu_{2}\right|\right)^{2}\left[\left(\left|\nu_{1}\right|+\left|\nu_{2}\right|\right)^{2}-1\right]} .
$$

Both Eqs. (4) and (5) are the predictions of the RMT on a quantum chaotic cavity, ${ }^{12}$ with the additional factors of 2 and 4 from the spin degeneracy. In the unipolar regime $\left(E_{f}^{(r 2)}\right.$ $>0$ ), plateaus of the ensemble average $\langle T\rangle$ are only partly formed when $\Delta=2 t$, and the height may not be accurate as the expected values given by 


$$
\langle T\rangle=2 \min \left(\left|\nu_{1}\right|,\left|\nu_{2}\right|\right) .
$$

The transmission functions show large ensemble variance where $\langle T\rangle$ has a large deviation from Eq. (6). Decreased disorder strength in the junction interface, in contrast, leads to better-developed plateaus of $\langle T\rangle$ in the unipolar regime at the cost of losing the quantized values of $\langle T\rangle$ and $\operatorname{Var}(T)$ in the bipolar regime. This is presented as the dash lines in Fig. 2 with $\Delta=0.5 t$.

The plateaus described by Eq. (4) in the bipolar regime and described by Eq. (6) in the unipolar regime are the signature of the quantum Hall effect in a single graphene junction, ${ }^{7}$ although in some cases the accuracy of the plateau is poor in the experimental data ${ }^{7,8}$ compared with the expected value. By taking into account the disorder in the junction interface area and a self-averaging process in the measurement, our calculations clearly produce these plateaus. In addition, the lack of the accuracy of the plateau height is attached to the strength of the disorder and is reflected in the variance of the transmission functions.

The experimentally observed conductance plateaus of a bipolar graphene junction in the quantum Hall regime have been explained as the result of the complete mixing of quantum Hall edge states at the junction interface due to scattering, and the departures of the experimental data from Eq. (4) have been attributed to the incomplete mixing of edge states. ${ }^{7,9}$ We emphasize here that because the spin-flip process is negligible in this system, the mode mixing can only happen among states of the same spin quantum number. Thus to correctly express $\langle T\rangle$ and $\operatorname{Var}(T)$ in terms of the filling factors $\nu_{1}$ and $\nu_{2}$, the filling factors must be spinless, with the spin degree of freedom included as an extra multiplier to $T$. Such expressions, compared with the expressions using the spinful filling factors $\pm 2, \pm 6, \pm 10 \ldots, 9$ show no quantitative difference as for $\langle T\rangle$ but significant differences as for $\operatorname{Var}(T)$.

The disorder dependence of the transmission functions with specific combinations of filling factors in the bipolar regime is shown in Fig. 3. The shadowed region (roughly $1.7 t<\Delta<2.8 t$ ) is where complete mode mixing happens in all three cases, i.e., $\nu_{1}=1$ and $\nu_{2}=-1,-3,-5$, respectively. The averaged transmission functions develop into plateaus of height described by Eq. (4) simultaneously in this region, and the ensemble variances of the transmission functions also develop into plateaus predicted by Eq. (5). In the language of the RMT, ${ }^{11,12}$ the ensembles of scattering matrices $S$ (of a specific spin quantum number) under these circumstances are the circular unitary ensembles (CUEs); that is, $S$ matrices in these ensembles are uniformly distributed over the unitary group $\mathcal{U}\left(\nu_{\text {total }}\right)$. The average over the CUE is equal to an integration over the unitary group. It is this integration that leads to the "universal" value of the averaged transmission function (4) and the universal conductance fluctuation (UCF) given by Eq. (5). The graphene bipolar junctions in this disorder regime (shadowed) are nearly ideal realizations of the quantum chaotic cavities characterized by Eqs. (4) and (5).

The ensemble of $S$ matrices, however, is actually dependent on the disorder strength $\Delta$, which represents how much

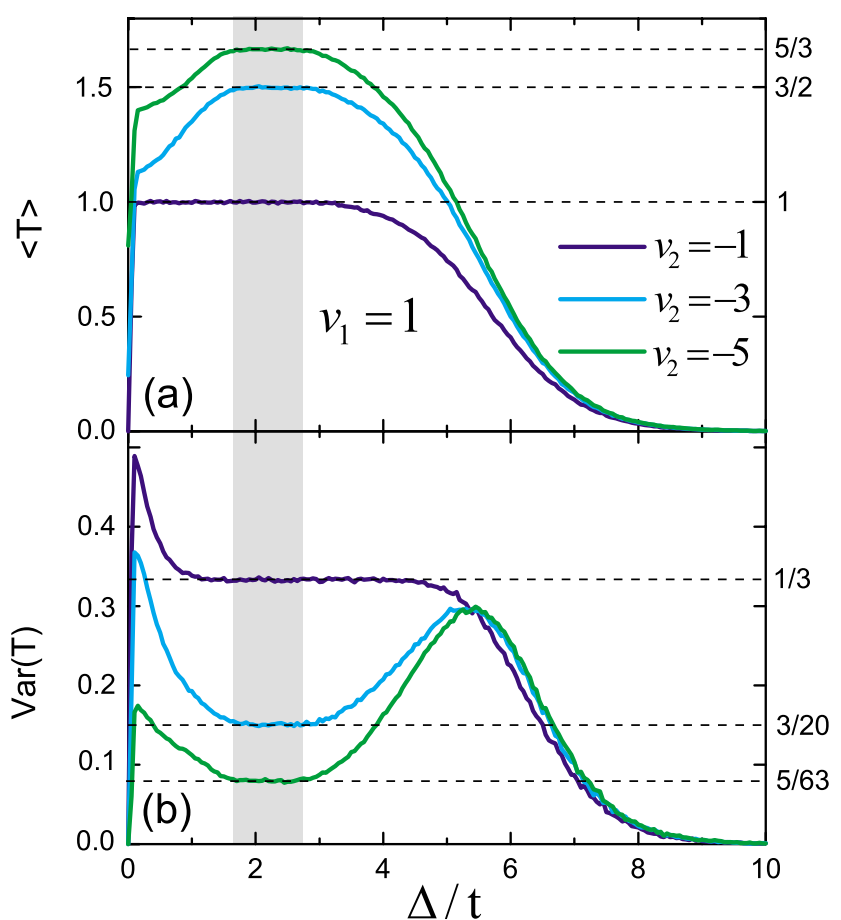

FIG. 3. (Color online) (a) The mean and (b) the variance of the transmission function $T$ as functions of the disorder strength $\Delta$ in bipolar junctions. Region 1 is of $n$ type with the filling factor $\nu_{1}$ $=1$ and region 2 is of $p$ type with the filling factor $\nu_{2}=-1,-3,-5$, respectively. The shadow highlights the regime where both $\langle T\rangle$ and $\operatorname{Var}(T)$ show plateaus predicted by Eqs. (4) and (5), respectively.

the scattering potential can be varied from one sample to another. In Fig. 3 we see that to the left of the shadowed region (roughly $\Delta<1.7 t$ ), both $\langle T\rangle$ and $\operatorname{Var}(T)$ deviate from Eqs. (4) and (5) with decreased $\Delta$, indicating the deviation of the actual ensembles of $S$ matrices from the CUEs. In other words, these are the cases of incomplete mode mixing. Notably, when $\nu_{1}=-\nu_{2}=1$ there is an extended range of $\Delta$ where the plateau of $\langle T\rangle$ is preserved. This is easily understood as there are only two modes to be mixed in this case. When the disorder at the junction interface is larger than the range of the shadowed region in Fig. 3, on the other hand, the conducting edge modes in either region 1 or region 2 are expelled from the junction interface area gradually. Scattering between modes from one side of the junction to the other is weak and both $\langle T\rangle$ and $\operatorname{Var}(T)$ decrease until the whole junction is "cut off." This is the case of what we see in the large- $\Delta$ regime of Fig. 3.

As compared with the bipolar junctions, transport in a unipolar junction with disordered junction interface has a more straightforward picture. The conductance of a unipolar junction is mainly contributed from the compatible edge modes in the two regions, and the tunneling of carriers from one region to another mainly happens near the lateral edges. The disorder at the interface impedes the coupling of these modes to reduce the transmission through the junction, which is in contrary to the bipolar cases. The calculated $\langle T\rangle$ and $\operatorname{Var}(T)$ in the unipolar regime are shown in Fig. 4 with different combinations of filling factors. In each case $\langle T\rangle$ exhibits a quantized plateau when the disorder is weak (shad- 


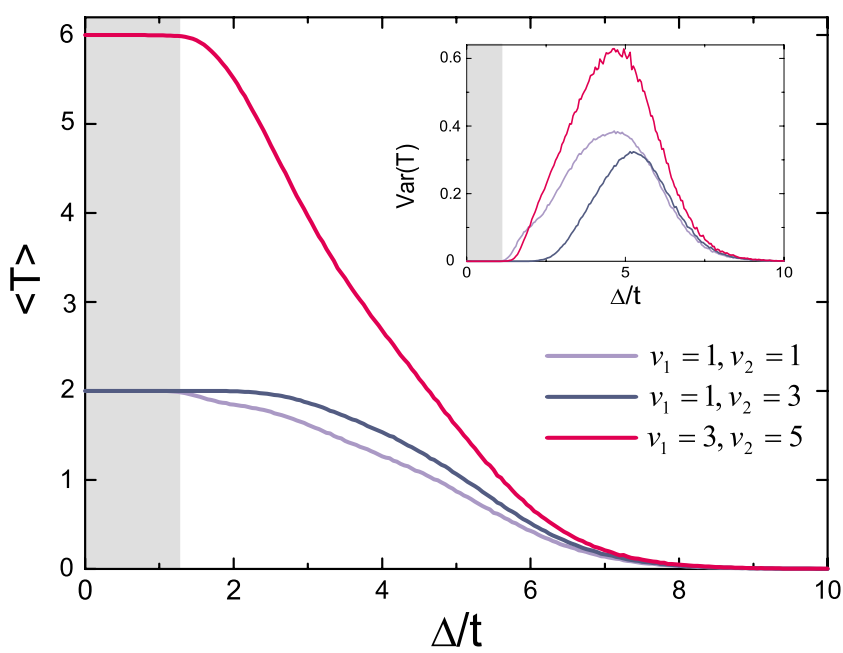

FIG. 4. (Color online) The averaged transmission function $\langle T\rangle$ as a function of the disorder strength $\Delta$ in unipolar $(n-n)$ junctions with different combinations of filling factors. The inset shows the corresponding variance of $T$. The regime is shadowed where all plateaus of $\langle T\rangle$ are preserved.

owed) and starts to deviate from the plateau at a critical value of the disorder strength where $\operatorname{Var}(T)$ also begins to deviate from zero. We notice that the shadowed regions in Figs. 3 and 4 do not overlap over the range of $\Delta$, which implies that $\langle T\rangle$ does not develop into plateaus simultaneously in the bipolar regime and the unipolar regime especially when the filling factors are large. This fact highlights the opposite roles the disorder plays in the formation of conductance plateaus in the bipolar and the unipolar junctions. It stabilizes the plateaus in the bipolar case when its intensity is in a certain nonzero range, while it tends to destroy the plateaus in the unipolar case at the same time, although in the limit of strong disorder electrons will be blocked from tunneling through the junction in both cases.

The character of the quantized transport in these junctions can be experimentally identified by measuring the electron shot noise. ${ }^{21}$ The Fano factor, defined as the ratio of the actual shot noise to the Poisson noise, is extracted from our simulation using the equation $F=\left\langle\Sigma T_{n}\left(1-T_{n}\right)\right\rangle /\left\langle\Sigma T_{n}\right\rangle$, where the summations are taken over different transmission eigenvalues indexed by $n$. It is found that the Fano factors corresponding to the conductance plateaus in the unipolar regime are identically zero as expected from the dissipationless transport via the quantum Hall edge modes. In the bipolar regime, the Fano factors develop into plateaus described by

$$
F=\frac{\left|\nu_{1} \nu_{2}\right|}{\left(\left|\nu_{1}\right|+\left|\nu_{2}\right|\right)^{2}-1}
$$

corresponding to the plateaus of $\langle T\rangle$, as shown in Fig. 5. Equation (7) is again a straightforward outcome of the RMT applied to a quantum chaotic cavity with a few transmission modes. ${ }^{22}$ It is quantitatively different from the Fano factors discussed in Ref. 9 especially when the number of the transmission modes is small. This is a key to examine experimentally the existence of the UCF revealed in our simulation.

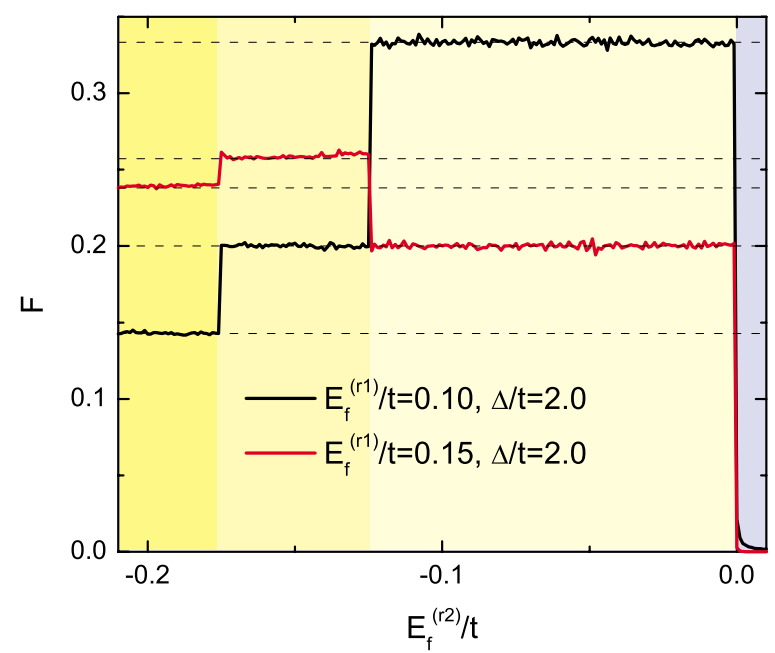

FIG. 5. (Color online) Fano factors in the bipolar regime as functions of the relative Fermi energy $E_{f}^{(r 2)}$ with $\nu_{1}=1$ (black) and 3 (red/dark gray), respectively, and $\Delta=2 t$. Plateaus predicted by Eq. (7) are indicated by short dash lines.

\section{DISCUSSIONS AND CONCLUSION}

Before ending this paper we address the issues on the sources and types of disorder in graphene, which are relevant to this study. The experimentally observed conductance plateaus were explained as a result of the full mixing of these quantum Hall edge modes. ${ }^{7,9}$ The details of the mode mixing are determined by the form of the disorder and in turn provide information about the disorder therein. The quantum Hall edge modes in a graphene strip can be indexed by two quantum numbers, spin and valley isospin, ${ }^{23}$ besides the Landau levels they belong to. Thus the mechanism of the mode mixing inevitably involves the presence of spin-flip scattering and/or intervalley scattering. Considering the negligible magnetic impurities and spin-related interaction in the current graphene samples and ignoring the magnetic edge states reported in some specific graphene ribbons, ${ }^{24}$ we assume the absence of spin-flip scattering in this study and use the spinless filling factors $\nu_{1}$ and $\nu_{2}(= \pm 1, \pm 3, \pm 5 \ldots)$ in our expressions. ${ }^{25}$ The presence of intervalley scattering among the quantum Hall edge modes in the graphene $p-n$ junctions, however, is still a question. Previous works on the disorder effects in graphene $p$ - $n$ junctions generally assumed weak intervalley scattering and discussed the valley-isospin dependence of the conductance. ${ }^{15}$ For example, in the work by Tworzydło et al. ${ }^{26}$ it was assumed that each impurity has the Gaussian potential profile $U_{i} \exp \left(-\left|\boldsymbol{r}-\boldsymbol{R}_{i}\right|^{2} / 2 \xi^{2}\right)$ of range $\xi$ and random height $U_{i} \in(-\delta, \delta)$. A large $\xi$ implies a long range of the disorder potential that suppresses the intervalley scattering, while a small $\xi$ implies $\delta$-functionlike disorder potential, which provokes the intervalley scattering. The former case was studied but the plateaus observed in the experiments were not properly recovered. That is the reason why we choose the latter case as our starting point in this work, although the origin of the short-range disorder potential is not completely understood. It turns out that our calculations do produce the reported conductance plateaus as in Eq. (4) as well as quantities such as the Fano factors as in 
Eq. (7) that can be further examined experimentally. Still it is interesting to investigate the crossover effects between longrange disorder potential and short-range disorder potential in the junctions, and the results would possibly explain the notfully-developed conductance plateaus in the experiments. ${ }^{7,8}$ This investigation will be a subject of our later work. Furthermore, there also exist other forms of disorder that cannot be described by random local potential. ${ }^{4,16}$ For example, effective gauge fields can be induced by ripples, topological lattice defects, strains, curvatures, etc. in the graphene sample and will affect the Landau levels especially the lowest one. ${ }^{27}$ We believe that these various forms of disorder will affect the behavior of the edge-mode mixing in various manners, but this is out of the scope of the current work.

In short, our numerical simulation of the quantum Hall effect in graphene $p$ - $n$ junctions has reproduced the quantized conductance plateaus observed in the experiment. The UCF and quantized values of the Fano factors are found to be accompanying the conductance plateaus in the bipolar regime, which is well explained by the RMT of quantum transport. The bipolar graphene junction in the quantum Hall regime mimics an ideal quantum chaotic cavity, which is another example of the extraordinary transport character of graphene.

Note added. At this point, we would like to note that we recently became aware of a similar work by W. Long et al. ${ }^{31}$

\section{ACKNOWLEDGMENT}

This work was supported by the Research Grant Council of Hong Kong under Grant No. HKU 7041/07P.

\section{APPENDIX: DERIVATION OF EQS. (4), (5), and (7)}

In this appendix, we derive Eqs. (4), (5), and (7) using the random matrix theory. Random matrix theory of quantum transport is based on the assumption that the scattering matrix $S$ of a chaotic cavity is uniformly distributed over a specific group (the so-called circular ensemble) determined by the symmetry of the Hamiltonian. Basically there are three classes of the groups. ${ }^{28}$ If time-reversal symmetry is broken $(\beta=2), S$ is only constrained by unitarity, which is a result of current conservation; thus, $S$ belongs to a unitary group. If time-reversal symmetry is preserved together with the presence of spin-rotation symmetry $(\beta=1)$, then $S$ is both unitary and symmetric: $S=S^{T}$, where the superscript $T$ indicates the transpose of the matrix. This leads to an orthogonal group. If time-reversal symmetry is preserved but spinrotation symmetry is broken $(\beta=4)$, which is the case when spin-orbit interaction is present, then $S$ is unitary and selfdual: $S=S^{R}$, where the superscript $R$ indicates the dual of a quaternion matrix. The group is called symplectic.

In random matrix theory, the statistics of transport properties is obtained from the statistics of an appropriate circular ensemble. For example, the mean of the transmission probability $T_{n m} \equiv\left|S_{n m}\right|^{2}$ is given by

$$
\left\langle T_{n m}\right\rangle=\int d \mu(S) S_{n m} S_{n m}^{*},
$$

where $n$ and $m$ stand for transmission eigenchannels. We will omit the mathematical details of formulating the measure $d \mu(S)$ in a group space where $S$ belongs to and use the following two equations as being established: ${ }^{29}$

$$
\left\langle U_{\alpha a} U_{\beta b}^{*}\right\rangle_{\mathrm{CUE}}=\frac{1}{N} \delta_{\alpha \beta} \delta_{a b},
$$

$$
\left\langle U_{\alpha a} U_{\alpha^{\prime} a^{\prime}} U_{\beta b}^{*} U_{\beta^{\prime} b^{\prime}}^{*}\right\rangle_{\mathrm{CUE}}=\frac{1}{N^{2}-1}\left(\delta_{\alpha \beta} \delta_{a b} \delta_{\alpha^{\prime} \beta^{\prime}} \delta_{a^{\prime} b^{\prime}}+\delta_{\alpha \beta^{\prime}} \delta_{a b^{\prime}} \delta_{\alpha^{\prime} \beta} \delta_{a^{\prime} b}\right)-\frac{1}{N\left(N^{2}-1\right)}\left(\delta_{\alpha \beta} \delta_{a b^{\prime}} \delta_{\alpha^{\prime} \beta^{\prime}} \delta_{a^{\prime} b}+\delta_{\alpha \beta^{\prime}} \delta_{a b} \delta_{\alpha^{\prime} \beta} \delta_{a^{\prime} b^{\prime}}\right)
$$

where $U$ is an $N \times N$ unitary matrix belonging to the CUE. Since the system we are going to discuss only involves the CUE, we will drop this notation hereafter.

Starting from these equations, the conductance $\langle G\rangle$, its fluctuation $\operatorname{Var}(G)$, and the Fano factor $F$ for the fully mixed quantum hall edge transport in a graphene $p$ - $n$ junction can be readily calculated. We first assume no spin-flip scattering is allowed, so that $S$ matrix for each spin component is unitary by itself. The case including spin-flip scattering will be discussed in what follows. Suppose there are $2 N_{1}$ and $2 N_{2}$ edge states (they are also eigenchannels away from the junction area) at the two sides of the junction, respectively, the full $S$ matrix is of dimension $2\left(N_{1}+N_{2}\right)$, but it is divided into two uncoupled spin subspaces in the absence of spin-flip scattering; therefore, matrices in the CUE are only of dimen- sion $N_{\text {total }}=N_{1}+N_{2}$ in terms of each spin subspace. Also the strict spin degeneracy implies that $S_{n m}^{\sigma}=S_{n m}^{-\sigma}=S_{n m}$. Regarding this we have

$$
\begin{aligned}
\langle G\rangle & =\sum_{\sigma= \pm 1} \sum_{n=1}^{N_{1}} \sum_{m=1}^{N_{2}}\left\langle T_{n m}^{\sigma}\right\rangle \frac{e^{2}}{h} \\
& =2 \sum_{n=1}^{N_{1}} \sum_{m=1}^{N_{2}}\left\langle S_{n m} S_{n m}^{*}\right\rangle \frac{e^{2}}{h} \\
& =\frac{2 N_{1} N_{2}}{N_{1}+N_{2}} \frac{e^{2}}{h},
\end{aligned}
$$




$$
\begin{aligned}
\operatorname{Var}(G) & =\left\langle G^{2}\right\rangle-\langle G\rangle^{2}=\left\langle\left(\sum_{\sigma= \pm 1} \sum_{n=1}^{N_{1}} \sum_{m=1}^{N_{2}} T_{n m}^{\sigma}\right)^{2}\right\rangle\left(\frac{e^{2}}{h}\right)^{2}-\langle G\rangle^{2} \\
& =4 \sum_{n, n^{\prime}=1}^{N_{1}} \sum_{m, m^{\prime}=1}^{N_{2}}\left\langle S_{n m^{\prime}} S_{n^{\prime} m^{\prime}} S_{n m^{*}}^{*} S_{n^{\prime} m^{\prime}}^{*}\right\rangle\left(\frac{e^{2}}{h}\right)^{2}-\langle G\rangle^{2} \\
& =\frac{4 N_{1}^{2} N_{2}^{2}}{\left(N_{1}+N_{2}\right)^{2}\left[\left(N_{1}+N_{2}\right)^{2}-1\right]}\left(\frac{e^{2}}{h}\right)^{2} .
\end{aligned}
$$

The Fano factor $F=\left\langle\operatorname{Tr}\left(t t^{\dagger}\left(1-t t^{\dagger}\right)\right)\right\rangle /\left\langle\operatorname{Tr}\left(t t^{\dagger}\right)\right\rangle$, where $t$ is the transmission submatrix of $S$ (Ref. 30) and $\operatorname{Tr}\left(t t^{\dagger}\right)$ is nothing but $G / \frac{e^{2}}{h}$. Thus

$$
\begin{aligned}
F & =1-\frac{N_{1}+N_{2}}{2 N_{1} N_{2}} \sum_{\sigma= \pm 1} \sum_{n, n^{\prime}=1}^{N_{1}} \sum_{m, m^{\prime}=1}^{N_{2}}\left\langle S_{n m}^{\sigma} S_{n^{\prime} m^{\prime}}^{\sigma *} S_{n^{\prime} m^{\prime}}^{\sigma} S_{n m^{\prime}}^{\sigma *}\right\rangle \\
& =\frac{N_{1} N_{2}}{\left(N_{1}+N_{2}\right)^{2}-1} .
\end{aligned}
$$

In this way we have derived the formulae expressed in Eqs. (4), (5), and (7) in the framework of the random matrix theory.

In contrast to the spin-conserved scattering case, the mixing among different spin modes will lead to quantitatively different variance and Fano factors while leaving the conductance mean unchanged. In the random matrix theory, this is derived as following. Still suppose there are $2 N_{1}$ and $2 N_{2}$ edge states at the two sides of the junction, respectively, the full $S$ matrix is still of dimension $2\left(N_{1}+N_{2}\right)$, but the spinoff-diagonal parts are now nonzero and the $S$ matrix is unitary only as a whole. It is straightforward to show that in this case the formulae corresponding to Eqs. (4), (5), and (7) are given by

$$
\langle G\rangle=\frac{4 N_{1} N_{2}}{2 N_{1}+2 N_{2}} \frac{e^{2}}{h}=\frac{2 N_{1} N_{2}}{N_{1}+N_{2}} \frac{e^{2}}{h},
$$

$$
\begin{aligned}
\operatorname{Var}(G) & =\frac{\left(4 N_{1} N_{2}\right)^{2}}{\left(2 N_{1}+2 N_{2}\right)^{2}\left[\left(2 N_{1}+2 N_{2}\right)^{2}-1\right]}\left(\frac{e^{2}}{h}\right)^{2} \\
& =\frac{4 N_{1}^{2} N_{2}^{2}}{\left(N_{1}+N_{2}\right)^{2}\left[\left(N_{1}+N_{2}\right)^{2}-1 / 4\right]}\left(\frac{e^{2}}{h}\right)^{2},
\end{aligned}
$$

and

$$
F=\frac{4 N_{1} N_{2}}{\left(2 N_{1}+2 N_{2}\right)^{2}-1}=\frac{N_{1} N_{2}}{\left(N_{1}+N_{2}\right)^{2}-1 / 4},
$$

respectively. Except for a coincident equivalence of the mean values of the conductance $\langle G\rangle$, the quantitative differences of the UCF $\operatorname{Var}(G)$ and the Fano factors $F$ between two cases, namely, whether scattering is spin conserved or not, are obvious especially when the values of $N_{1}$ and $N_{2}$ are small.
${ }^{1}$ K. S. Novoselov, A. K. Geim, S. V. Morozov, D. Jiang, Y. Zhang, S. V. Dubonos, I. V. Grigorieva, and A. A. Firsov, Science 306, 666 (2004); K. S. Novoselov, D. Jiang, F. Schedin, T. J. Booth, V. V. Khotkevich, S. M. Morozov, and A. K. Geim, Proc. Natl. Acad. Sci. U.S.A. 102, 10451 (2005).

${ }^{2}$ A. K. Geim and K. S. Novoselov, Nature Mater. 6, 183 (2007).

${ }^{3}$ M. I. Katsnelson and K. S. Novoselov, Solid State Commun. 143, 3 (2007).

${ }^{4}$ A. H. Castro Neto, F. Guinea, N. M. R. Peres, K. S. Novoselov, and A. K. Geim, arXiv:0709.1163, Rev. Mod. Phys. (to be published).

${ }^{5}$ K. S. Novoselov, A. K. Geim, S. V. Morozov, D. Jiang, M. I. Katsnelson, I. V. Grigorieva, S. V. Dubonos, and A. A. Firsov, Nature (London) 438, 197 (2005).

${ }^{6}$ Y. B. Zhang, Y. W. Tan, H. L. Stormer, and P. Kim, Nature (London) 438, 201 (2005).

${ }^{7}$ J. R. Williams, L. DiCarlo, and C. M. Marcus, Science 317, 638 (2007).

${ }^{8}$ B. Özyilmaz, P. Jarillo-Herrero, D. Efetov, D. A. Abanin, L. S. Levitov, and P. Kim, Phys. Rev. Lett. 99, 166804 (2007).

${ }^{9}$ D. A. Abanin and L. S. Levitov, Science 317, 641 (2007).

${ }^{10}$ R. Landauer, IBM J. Res. Dev. 1, 223 (1957); Philos. Mag. 21, 863 (1970); M. Büttiker, IBM J. Res. Dev. 32, 317 (1988); Phys. Rev. B 38, 9375 (1988).

${ }^{11}$ H. U. Baranger and P. A. Mello, Phys. Rev. Lett. 73, 142 (1994); R. A. Jalabert, J. L. Pichard, and C. W. J. Beenakker, Europhys. Lett. 27, 255 (1994).

${ }^{12}$ C. W. J. Beenakker, Rev. Mod. Phys. 69, 731 (1997).
${ }^{13}$ V. P. Gusynin and S. G. Sharapov, Phys. Rev. Lett. 95, 146801 (2005).

${ }^{14}$ See, for instance, Fig. 21 in the review by Castro Neto et al. (Ref. 4).

${ }^{15}$ V. V. Cheianov and V. I. Fal'ko, Phys. Rev. B 74, 041403(R) (2006); J. Tworzydło, I. Snyman, A. R. Akhmerov, and C. W. J. Beenakker, ibid. 76, 035411 (2007); A. V. Shytov, Nan Gu, and L. S. Levitov, arXiv:0708.3081 (unpublished).

${ }^{16}$ Vitor M. Pereira, J. M. B. Lopes dos Santos, and A. H. Castro Neto, Phys. Rev. B 77, 115109 (2008).

${ }^{17}$ L. Brey and H. A. Fertig, Phys. Rev. B 73, 195408 (2006).

${ }^{18}$ P. W. Anderson, Phys. Rev. 109, 1492 (1958).

${ }^{19}$ P. A. Lee and D. S. Fisher, Phys. Rev. Lett. 47, 882 (1981).

${ }^{20}$ J. Li, L. B. Hu, and S. Q. Shen, Phys. Rev. B 71, 241305(R) (2005); J. Li and S. Q. Shen, ibid. 76, 153302 (2007).

${ }^{21}$ Y. M. Blanter and M. Buttiker, Phys. Rep. 336, 1 (2000).

${ }^{22}$ D. V. Savin and H. J. Sommers, Phys. Rev. B 73, 081307(R) (2006).

${ }^{23}$ A. R. Akhmerov and C. W. J. Beenakker, Phys. Rev. Lett. 98, 157003 (2007).

${ }^{24}$ Y. W. Son, M. L. Cohen, and S. G. Louie, Nature (London) 444, 347 (2006).

${ }^{25} \mathrm{By}$ the random matrix theory, the calculations including or excluding mixing of different spin modes lead to the same expression for the averaged conductance, but with different expressions for the conductance fluctuation and the Fano factor, which is derived in the Appendix. This accounts for the differences between Eqs. (5) and (7) in this work and Eqs. (4) and (10) in the work by Abanin and Levitov (Ref. 9). 
${ }^{26}$ J. Tworzydło, I. Snyman, A. R. Akhmerov, and C. W. J. Beenakker, Phys. Rev. B 76, 035411 (2007).

${ }^{27}$ F. Guinea, B. Horovitz, and P. Le Doussal, Phys. Rev. B 77, 205421 (2008).

${ }^{28}$ F. J. Dyson, J. Math. Phys. 3, 1199 (1962).

${ }^{29}$ M. Creutz, J. Math. Phys. 19, 2043 (1978); S. Samuel, ibid. 21,
2695 (1980).

${ }^{30}$ In general $S$ can be written in terms of its transmission and reflection parts as $S=\left(\begin{array}{c}r t^{\prime} \\ t t^{\prime}\end{array}\right)$

${ }^{31}$ W. Long, Q. F. Sun, and J. Wang, Phys. Rev. Lett. 101, 166806 (2008). 